\title{
Von der Leyens fünf Heraus- forderungen für ein besseres Europa
}

So richtig glücklich war am Ende niemand über die Wahl von Ursula von der Leyen zur Kommissionspräsidentin. Das lag aber weniger an ihr als vielmehr an dem Prozess bis zu ihrer Ernennung. Dass sich zumindest in dieser Kritik fast alle politischen Parteienfamilien im Europäischen Parlament einig sind, könnte sich als entscheidende Stärke in den kommenden fünf Jahren herausstellen. Denn die Europäische Union (EU) steht vor Herausforderungen, die mutige Reformen über Parteigrenzen hinweg erfordern. Und die Vorschläge von der Leyens, wenn auch wenig detailliert, zeugen von dem Mut, Tabus zu brechen und die Ziele hoch zu stecken.

Europa steht an einem Wendepunkt, vielleicht dem wichtigsten der vergangenen 50 Jahre. Die EU ist großen internen Fliehkräften ausgesetzt: Großbritannien wird die EU wohl verlassen, die italienische und andere Regierungen lassen sich von populistischen Kräften vereinnahmen und fast überall in Europa nimmt der Nationalismus zu. Extern muss sich Europa der Bedrohung durch den zunehmenden Nationalismus und Protektionismus der USA und Chinas erwehren und sich in einem intensiver werdenden Systemwettbewerb behaupten. US-Präsident Donald Trump geht auf aggressiven Konfrontationskurs zu Europa, das in seinen Augen schwach und führungslos ist. China versucht, seine Interessen etwas subtiler durchzusetzen: durch bilaterale Abkommen mit Regierungen in Zentral- und Osteuropa und eine gezielte Wirtschaftspolitik. Die Gefahr ist groß, dass ein uneiniges Europa in diesem Systemwettbewerb nicht bestehen wird und die wichtigen globalen Entscheidungen nur noch bilateral zwischen den USA und China getroffen werden.

Sowohl die internen als auch die externen Herausforderungen machen grundlegende Reformen und eine konzertierte Antwort Europas nötig. Dazu bedarf es einer starken EU-Kommission, die zwar auch eine politische Rolle hat, aber mehr als unter Kommissionspräsident Jean-Claude Juncker eine starke inhaltliche Führungsrolle in Europa übernehmen muss. Ursula von der Leyen mag für diese Aufgabe sehr viel besser geeignet sein, als viele Kritiker meinen. Sie hat als Ministerin über 14 Jahre mit den Portfolios Familie, Arbeit und Soziales und zuletzt Verteidigung eine Breite an Erfahrung gesammelt, die kaum ein anderer Politiker in Europa nachweisen kann. Dabei hat von der Leyen klar signalisiert, dass sie unabhängig und glaubwürdig im Sinne ganz Europas handeln wird, und nicht davor zurückschreckt, als Kommissionspräsidentin auch deutsche Tabus zu brechen.

Die neue EU-Kommission muss fünf zentrale Herausforderungen angehen. Die erste Herausforderung ist die Stärkung öffentlicher Güter in Europa. Dazu gehört eine gemeinsame Lösung bei der Lastenverteilung und Verantwortung bei Migration und Flucht, aber auch eine stärkere gemeinsame Sicherheits- und Außenpolitik. Von der Leyens Forderung nach einer „Armee der Europäer“, die die Verantwortung nationaler und europäischer Ebenen bündelt, wäre eine kluge Lösung, die einen breiten Konsens ermöglicht. Auch ihre pragmatische Position gegenüber Großbritannien zeigt den richtigen Instinkt, eine enge Verbindung auch nach dem Brexit beizubehalten.

Die zweite große Priorität muss die Vollendung des Binnenmarkts und der Währungsunion sein. Will Europa in Zukunft im globalen Konzert mit den USA und China mitspielen, dann braucht es eine starke globale Rolle des Euro, einen integrierten Kapitalmarkt, eine Reform des Binnenmarkts für Dienstleistungen und stärkere gemeinsame Regeln. Von der Leyen hat die richtigen Prioritäten gesetzt: mehr Flexibilität in der Finanzpolitik

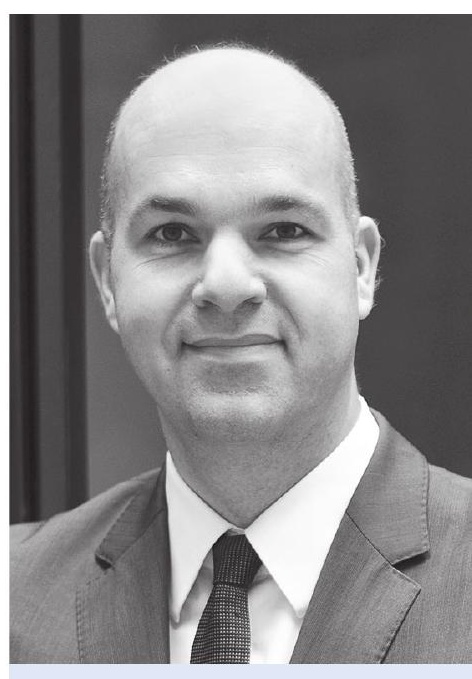

Marcel Fratzscher ist Hochschullehrer an der Humboldt-Universität zu Berlin und Präsident des Deutschen Instituts für Wirtschaftsforschung (DIW) in Berlin. 
und bei den Schulden, eine Rückversicherung für Arbeitslose und die Vollendung der Bankenunion mit einer Einlagensicherung. Sie hat damit wohl bewusst rote Linien der Bundesregierung und vor allem der CDU überschritten. Der Euro kann nur sinnvoll reformiert werden, wenn der deutsche Fokus auf Regeln, Märkte und Risikoreduktion mit dem anderer auf Flexibilität, staatliche Regulierung und Solidarität einhergeht. Frankreich und andere müssen also genauso kompromissbereit sein wie Deutschland. Aber auch in der globalen Wirtschafts- und Finanzpolitik muss sie in Zukunft mehr Verantwortung übernehmen. Die EU hat in den letzten Jahren bewiesen - durch Freihandelsabkommen mit Kanada, Japan und Südamerika (Mercosur) bis hin zum Pariser Klimaabkommen -, dass sie durchaus in der Lage ist, eine stärkere globale Verantwortung zu übernehmen. Dazu muss die EU in globalen Foren, wie dem UN-Sicherheitsrat, und internationalen Organisationen, wie dem Internationalen Währungsfonds (IWF) oder der Weltbank, in Zukunft mit einer und nicht mit vielen Stimmen sprechen.

Die dritte Priorität liegt im Bereich der Nachhaltigkeit und Klimapolitik. Von der Leyens Ziel, Europa müsse bis 2050 klimaneutral sein, halten viele für unrealistisch und überzogen. Es ist aber wichtig für die EU-Kommission, eine eigene Vision für Europa zu entwickeln und zu verfolgen. Und was heißt unrealistisch? Jacques Delors, der 1985 EUKommissionspräsident wurde, ist anfänglich für seine Vision einer gemeinsamen Währung belächelt worden. Aber seine Arbeit - zusammen mit Helmut Kohl und François Mitterrand - war mit entscheidend, um den Euro 1999 Realität werden zu lassen. Und das Ziel der Klimaneutralität bis 2050 ist nicht nur realistisch, sondern alternativlos.

Ein stärkeres soziales Europa sollte die vierte Priorität der neuen EU-Kommission sein. Nicht nur der Euro als gemeinsame Währung, sondern die EU als Ganzes kann nur dann nachhaltig funktionieren, wenn es einen breiten Konvergenzprozess gibt, also ein wirtschaftliches und soziales Zusammenwachsen Europas. Ein gemeinsames Budget für Konvergenz und Wettbewerbsfähigkeit ist ein erster Schritt, aber bei weitem nicht ausreichend - genauso wie Mindestlöhne in ganz Europa und Einzelmaßnahmen zur Bekämpfung der Jugendarbeitslosigkeit. Um eine solche Konvergenz zu erzielen, müsste die neue EU-Kommission das bestehende EU-Budget drastisch umgestalten und die $80 \%$ des Budgets, die für Strukturhilfen und Subventionen für die Landwirtschaft ausgegeben werden, viel gezielter zur Unterstützung strukturell schwacher Regionen aufbringen. Ein Investitionsfonds als Nachfolge des JunckerPlans sollte geschaffen werden, um wichtige öffentliche Investitionsprojekte anzustoBen - von Digital- und Verkehrsinfrastruktur bis hin zu Forschung und Entwicklung.

Die fünfte und vielleicht wichtigste Priorität der neuen EU-Kommission sollten Reformen der europäischen Institutionen sein, mit dem Ziel, deren Legitimität und Glaubwürdigkeit zu stärken. Die EU ist zu einem perfekten Sündenbock für Nationalisten in Europa geworden, die über eine überbordende Brüsseler Bürokratie oder zu wenig nationale Souveränität klagen. Transnationale Listen und ein richtiger Spitzenkandidatenprozess würden es den Menschen ermöglichen, ihren Kommissionspräsidenten direkt zu wählen, und so auch der EU-Kommission und dem europäischen Parlament mehr Legitimität verleihen. Auch der Bürgerdialog ab 2020, den von der Leyen vorschlägt, ist klug, um eine breite Debatte anzustoßen, wieso Europa für seine Bürger wichtig ist und was sie sich von den europäischen Institutionen erhoffen.

Europa stand in den letzten 70 Jahren selten vor so grundlegenden Herausforderungen wie heute. Die internen Fliehkräfte und die externen Bedrohungen durch China und die USA erfordern mutige Reformen mit fünf zentralen Prioritäten. Statt sich über

Marcel Fratzscher

Deutsches Institut für Wirtschaftsforschung mfratzscher@diw.de
Personalien zu streiten, sollte nun eine inhaltliche Debatte geführt und die Frage beantwortet werden, wie Europa wirtschaftlich, politisch und sozial reformiert werden kann, um auch global endlich seiner Verantwortung gerecht zu werden. 